\title{
Research on High-Speed Face Authentication Algorithm for IoT Devices
}

\author{
Naoya Shinozaki ${ }^{\mathrm{a}, *}$, Meng Ge ${ }^{\mathrm{a}}$, Akira Yamawaki, Seiichi Serikawa ${ }^{\mathrm{a}}$, Lifeng Zhang ${ }^{\mathrm{a}}$ \\ ${ }^{a}$ 1-1sensui-cho Tobataku, Kitakyushu city, Fukuoka Japan 804-8550 \\ Kyushu Institute of Technology \\ *Corresponding Author: shinozaki.naoya223@mail.kyutech.jp
}

\begin{abstract}
Face authentication is considered as the most convenient approach of biometric authentication techniques and has been put into practical use in various situations. But face has a significant feature that other biometrics do not have is it keeps changing lifelong. Even in the morning or at night, with the body conditions good or bad, face shows different appearance. Furthermore, the accuracy of face authentication is also affected by changes in facial expressions and face orientation. There are also many technical difficulties because the face authentication requires both compatibility of accuracy and speed, but it is difficult to implement complicated calculations into an impotent devices.

This study proposes a simple face authentication algorithm for IoT devices. Proposed method obtains a certain level of face authentication accuracy and high speed operation can be achieved at the same time.
\end{abstract}

Keywords: face authentication, high-speed, IoT device

\section{Introduction}

\subsection{Face Authentication}

With the recent informationization, the identity authentication is becoming important more and more. In the case of using ATM of a bank, even at the time of entering your home building, there are cases which people are need to be authenticated, and these cases will increase in the future.

There are several methods for identity authentication, such as possessed object authentication, intellectual authentication, biometric authentication, etc. Among them, it is difficult for a person to spoof the biometric authentication that utilizes the physical features of the person. We think that the biometric authentication is the most needed in the future society. In addition, face authentication is widely recognized in biometrics authentication.

Face authentication is a system for identifying a person from an input image. It is necessary to be registered in advance and the system makes a judgment whether the input face image is registered or not. And the task of the face authentication system is the speed and accuracy of authentication. If we make the algorithm complicated to improve accuracy, a computer must do complicated calculation, and as a result, the authentication speed will be down. In addition, impotent devices which have less processing capability cannot do complicated calculation. They cannot do complicated calculations or take a very long time.

Therefore, we tried to reduce computational complexity by simplifying algorithms and try to achieve both high speed and some degree of authentication precision even for impotent devices.

\section{$1.2 \quad$ IoT Devices}

IoT is an abbreviation for Internet of Things. By connecting various other things to the Internet like a personal computer, we can know the state of remote things and operate them. This idea will be able to improve our lives. The reason why attention to IoT is increasing is that the number of devices which can be connected to the Internet is increasing.

However, not all IoT devices are highly functional. There are many devices with poor performance such as low computing power. In such a device, it is difficult to implement a complicated algorithm for face authentication. And even if it is implemented, problems will arise in terms of its speed and accuracy. Therefore, we thought that the algorithm for such impotent devices is necessary.

As shown in Figure 1, the number of IoT devices continues to increase year by year. It is expected to exceed 50 billion by 2020 . 


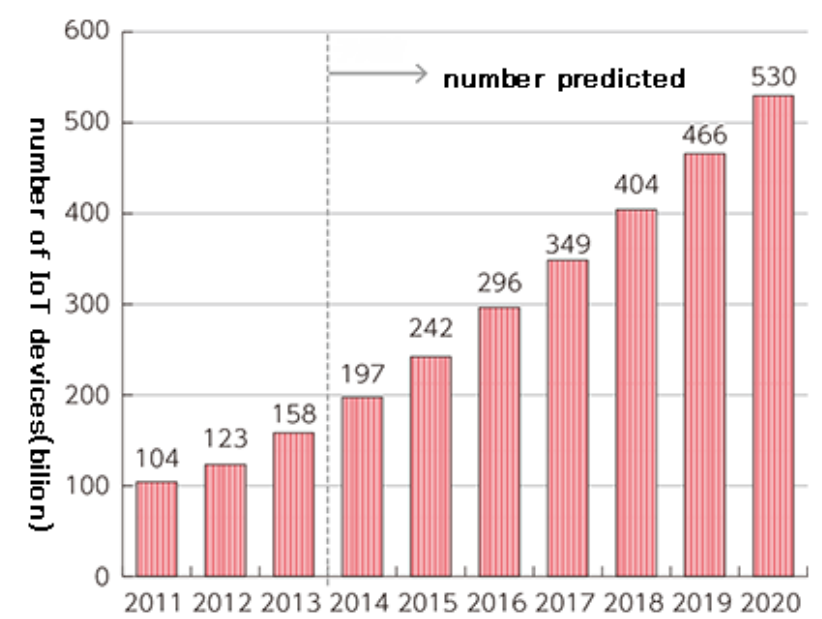

Fig. 1. Number of IoT Devices

(http://www.soumu.go.jp/johotsusintokei/whitepaper/ja/h27 /html/nc254110.html)

\subsection{Proposal}

For the implementation on IoT devices, we develop a simple algorithm that performs face authentication only with the sign of DCT coefficients, and investigate the performance of face authentication.

Specifically, we examine how the authentication rate varies with the number of points of DCT coefficients we take. And we examine how the authentication rate changes by the positions of DCT coefficients. We gradually shift DCT coefficients from the larger amplitude to the smaller amplitude. We investigate how many images are received when we input 100 images of registered person and when we input 100 images of other people.

\section{Principle}

\subsection{System prerequisites}

As explained in the previous section, the face authentication system is one of the biometrics authentication, and it is an application which can distinguish a person from the input image. The face is photographed in front of the camera, and using the part considered to be face, identification is done by comparing with the information registered in advance.

As described above, the face authentication system assumes that the face is not moved so much toward the front, and cannot cope with the case that it is not directed to the front, so the same assumption is assumed in this system. It is

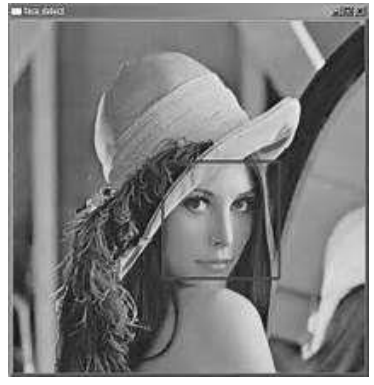

Fig. 2. Face Detection

not supposed to shake the face extremely up, down, left and right. Also, because of the nature of discrimination using only the sign of the DCT coefficients, it is difficult to discriminate too many people.

\subsection{Face Detection}

Face detection is a technique for determining where a face is in a given image. First of all, we get an image from the camera and convert it to grayscale. After that, face detection is performed and only the face area is cut out from the original image using the position coordinates of the face. After cutting out the face area, resize the face area of original image to a size of $128 \times 128$ pixels. This $128 \times 128$ pixels image is the registered image which we derive information for face authentication.

\subsection{DCT(Discrete Cosine Transform)}

After performing face detection, DCT is performed to achieve both face authentication accuracy and speed compatibility. DCT is a method of converting a discrete signal to a frequency domain. By sampling the image and converting it to a discrete signal, performing DCT and encoding. it is possible to reduce the data capacity without damaging the majority of the original signal.

The DCT is closely related to the Discrete Fourier Transform. DCT is one of the separable linear transformations. Therefore, the two-dimensional transformation corresponds to executing one-dimensional DCT along one dimension and then executing onedimensional DCT in another dimension. The definition of two-dimensional DCT for input image A and output image B is as follows.

$$
\begin{gathered}
\mathrm{B}_{\mathrm{pq}}=\alpha_{\mathrm{p} \alpha} \alpha_{q} \sum_{m=0}^{M-1} \sum_{n=0}^{N-1} A_{m n} \cos \frac{\pi(2 m+1) p}{2 M} \cos \frac{\pi(2 n+1) p}{2 N} \\
0 \leq \mathrm{p} \leq \mathrm{M}-1
\end{gathered}
$$




$$
\begin{gathered}
0 \leq \mathrm{q} \leq \mathrm{N}-1 \\
\alpha_{\mathrm{p}}=\left\{\begin{array}{cc}
\frac{1}{\sqrt{M}}, & p=0 \\
\sqrt{\frac{2}{M}}, & 1 \leq p \leq M-1
\end{array}\right. \\
\alpha_{q}=\left\{\begin{array}{cc}
\frac{1}{\sqrt{N}}, & q=0 \\
\sqrt{\frac{2}{N}}, & 1 \leq q \leq N-1
\end{array}\right.
\end{gathered}
$$

$\mathrm{M}$ and $\mathrm{N}$ are the row size and the column size of A. DCT has the feature that the converted frequency component concentrates in the low frequency region.

In face image recognition by DCT, we use the fact that the DCT coefficients are greatly different among individuals. In this study, slicing DCT coefficients with a certain threshold value, sorting in descending order of values, retrieving important information, and discarding other information as low importance. As a result, data capacity can be reduced and high-speed operation will be expected.

The image of DCT is shown in Fig.3

\section{Experimental Method}

The face image in this experiment is photographed with a web camera, and it is assumed that one image for registration and 100 images for authentication are input. Also, 100 images of the same person that is registered and 300 images of different three people were prepared for authentication images. The input image satisfies the following conditions.

- A human face exists in the image

- Color image

- A person do not face extremely sideways to the camera or face up and down

- A person is not approaching or getting too far from
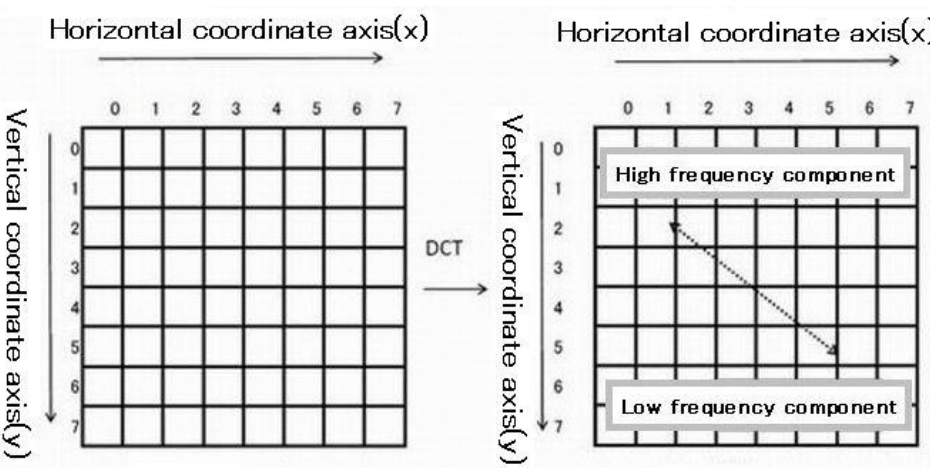

Fig. 3. Image of DCT the camera

We developed a face authentication system that operates at high speed with the contents described in the previous section, and actually verified it. The size of the input image is $640 \times 480$ pixels, and after cutting out the face area, resize it to $128 \times 128$ pixels and make other input image all the same size. In addition, the DCT coefficients are examined for two patterns, a pattern to extract 64 points in the low frequency region and a pattern to extract 32 points. It is checked what percentage of the extracted points coincide with each other.

In this case, since face authentication is performed only by whether or not the plus and minus signs match, it is considered that about $50 \%$ coincidentally coincides. Therefore, it is dangerous to declare that the input person is the same person that is registered even if, for example, $80 \%$ signs coincide. In this experiment, we judged that it is the same person when the signs match $90 \%$ or more. We also shifted DCT coefficients from the larger amplitude to the smaller amplitude by 5 points, and examined how the performance of face authentication changes by the way which DCT coefficients are taken. After sorting in descending order of the DCT coefficients, experiments are performed by changing the point used for authentication such that the first to the 64th are extracted, next the sixth to the 69 th are extracted. Shift of the points to be used like this is done five times.

Its face of the image for registration faces the front of the camera and there is no change in facial expression. The image for authentication is moving the face a little or

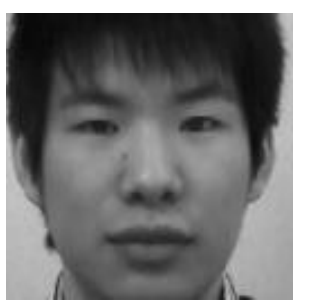

(a) A's registration image

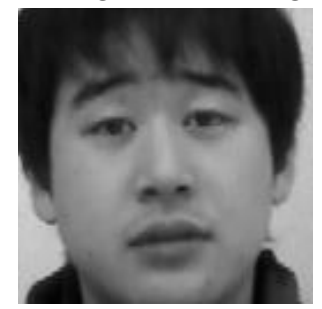

(c) C's registration image

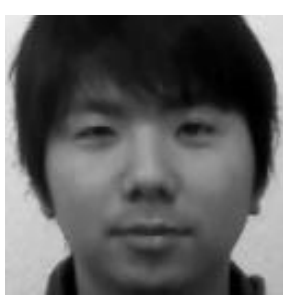

(b) B's registration image

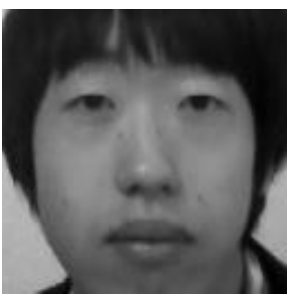

(d) D's registration image
Fig. 4. Each registration image 
changing the expression so that only the same image will be input. The images used for registering persons A, B, C and $\mathrm{D}$ are shown in Fig.4.

The configuration of the personal computer used in this experiment is shown below.

- OS: Windows10

- Processor: Core i3-6100

- Operating frequency: $3.70 \mathrm{GHz}$

- Memory: $4.00 \mathrm{~GB}$

We selected Visual Studio 2015 as an execution environment.

\section{Results}

\subsection{Authentication Speed}

Throughout the experiment, the authentication speed

Table 1. Number of facial images authenticated per 100(using 64 points for authentication)

\begin{tabular}{|c|c|c|}
\hline $\begin{array}{c}\text { Position of the } \\
\text { point }\end{array}$ & $\begin{array}{c}\text { Acceptance } \\
\text { number of } \\
\text { principals }\end{array}$ & $\begin{array}{c}\text { Acceptance } \\
\text { number of } \\
\text { others }\end{array}$ \\
\hline $1-64$ & 36.00 & 1.250 \\
\hline $6-69$ & 28.75 & 0.500 \\
\hline $11-74$ & 23.25 & 0 \\
\hline $16-79$ & 18.75 & 0 \\
\hline $21-84$ & 18.00 & 0 \\
\hline $26-89$ & 15.75 & 0 \\
\hline
\end{tabular}

Table 2. Number of facial images authenticated per 100(using 32 points for authentication)

\begin{tabular}{|c|c|c|}
\hline $\begin{array}{c}\text { Position of the } \\
\text { point }\end{array}$ & $\begin{array}{c}\text { Acceptance } \\
\text { number of } \\
\text { principals }\end{array}$ & $\begin{array}{c}\text { Acceptance } \\
\text { number of } \\
\text { others }\end{array}$ \\
\hline $1-32$ & 54.50 & 19.92 \\
\hline $6-37$ & 43.50 & 13.50 \\
\hline $11-42$ & 38.25 & 7.583 \\
\hline $16-47$ & 32.00 & 2.750 \\
\hline $21-52$ & 26.00 & 1.167 \\
\hline $26-57$ & 25.25 & 0.6667 \\
\hline
\end{tabular}

was on average of 30 milliseconds per image. Assuming that the maximum frame rate of the camera is $30 \mathrm{fps}$, it is possible to perform face authentication for almost all frames.

\subsection{Authentication Rate}

(1) In case taking 64 DCT coefficients

We took 64 DCT coefficient signs and shift the offset of the selection in large order of amplitude five times by 5 points per operation. Then investigated how many images are certified for 100 different images of the same person.

The number of facial images authenticated for 100 different images of the same person is shown in Table 1. As shown in Table 1, the acceptance number of principals is down when we shift the points used for authentication. And the acceptance of number of others is also down. We obtained the result that taking the $11^{\text {th }}$ to $74^{\text {th }}$ points has the best authentication rate.

(2) In case taking 32 DCT coefficients

We took 32 DCT coefficients signs and shift the offset of the selection in larger order of amplitude five times by 5 points per operation. Then investigated how many images are certified for 100 different images of the same person.

The number of facial images authenticated 100 different images of the same person is shown in Table 2. As shown in Table2, the acceptance number of principals and acceptance number of others increased compared to using the 64 points. And the acceptance number of principals and acceptance number of others are down like authentication using 64 points when we shift the points used. But in this experiment erroneous authentication did not become zero.

\section{Conclusion}

In this Study, we performed face authentication using only the sign of DCT coefficients, and examined how the performance changes by the number of points to be taken and the position of the points to be used. As for the authentication speed, 30 milliseconds per facial image is thought sufficient. Next, regarding the authentication rate, it can be seen that it varies greatly depending on the number and order of DCT coefficients. When 64 DCT coefficients were taken, the FRR(False Rejection Rate) increased more than when 32 points were taken. And as we shift the DCT signs which we take, we can see that the FAR(False Acceptation Rate) will 
decrease, both of person himself and others.

As a result, it was found that it is possible to distinguish only by the sign of the DCT coefficient. We thought that it is possible to realize a simple face authentication system which can work at high speed on IoT devices by using this method.

However, there are still many points need to improve. Speed and authentication rate are expected to be improved more than now, devising how to select feature value and how to calculate. We are planning to automatically decides how many DCT coefficients to take and which position to take by machine learning. By doing this, the FAR is expect to decline. It will be more useful as a face authentication system. Based on the results obtained in this study, we will raise the quality of the face authentication system with a simple algorithm, but the number and the position of points best suited for authentication are not clear yet, we have to investigate it. And we did these experiments on the general computer, so we must investigate the authentication quality when we implement this algorithm on the IoT devices.

\section{References}

(1) Koichi Sakai: "Introduction to image processing and pattern recognition: from the basics until project creation by VC \# / VC ++. NET”, pp. 101-112, 2006

(2) Hiroshi Nagahashi: "Image analysis theory(3)", http://www.isl.titech.ac.jp/nagahashilab/member/longb/ imageanalysis/LectureNotes/ImageAnalysis03.pdf

(3) Kazuhiko Sumi etc:" Personal authentication technology that has improved accuracy", pp137-155, 2014

(4) Masaaki Mitani: "Signal mathematics for studying again”, pp257-378, 2004 